\title{
Teaching Digital Circuit Design to Middle-School Students
}

\section{Dr. Christopher R. Carroll, University of Minnesota Duluth}

Dr. Carroll received his undergraduate education at Georgia Tech, and received the MSEE and PhD in Computer Science degrees from Caltech. He is currently Associate Professor of Electrical Engineering at the University of Minnesota Duluth. His interests include special purpose digital system design, VLSI, and microprocessor systems, especially as they relate to educational environments. 


\title{
Teaching Digital Circuit Design to Middle-School Students
}

\begin{abstract}
During the summer of 2012, the Electrical Engineering department at the University of Minnesota Duluth offered a summer camp for seventh-grade students to attempt to interest them in engineering. Among the topics included in this one-week summer camp was a one-day exposure to digital circuit design. How does one teach digital circuit design in one day to students who are in the midst of summer vacation and who have no technical background? What are the vital basics? What is unnecessary confusion? How can seventh-graders be entertained while still inspiring them to consider engineering concepts? These questions were among those faced by the Electrical Engineering faculty. This paper exposes some of the techniques used to intrigue students and to capture their interest in digital circuit design.
\end{abstract}

Attracting middle-school students to the field of engineering is a challenge. Students' interest must be captured before societal pressures deflect career choices into other directions. Students, must be encouraged to consider engineering as an interesting field before it is "too late" to enter the necessary math and science tracks in high school. Consequently, students must be exposed to the excitement and potential of engineering during their middle-school education to motivate them successfully to consider engineering careers. The goal of the summer camp was to dazzle students with fun applications that engineering makes possible, without intimidating them with undue complexity or technical details.

Topics presented to these seventh-graders during their one-day exposure to digital circuit design included both combinational and sequential circuits, although only in very restricted arenas. Only two different types of digital components were described, NAND gates and D-flip-flops. A seven-segment LED display digit was used to show the output produced by student circuits because these students were familiar with such displays from their everyday life. Students performed a variety of experiments from simply making a light flash to creating custom patterns on the seven-segment display, with a wide range of success. Overall, students seemed to enjoy the experience.

This paper describes the topics presented to these middle-school students in digital circuit design, and details some "lab experiments" performed to provide hands-on experience. Also included are lessons learned by the Electrical Engineering faculty to improve future camp offerings.

\section{Zeros and Ones}

This one-day exposure to digital circuits began with "What's Digital?" and "What's a Circuit?" questions. These students had heard the word digital throughout their lives and associated it with technical advances and modern implementations. What they learned in this camp is that digital means that variables can have one of only a fixed number of values, typically just two values, conveniently called $\mathbf{0}$ and $\mathbf{1}$. That's enough information to distinguish digital things from the real-world, continuous experience with which they had daily exposure. Digital does not mean 
that digits are involved, but rather means that variables interact under very different and limited rules compared to familiar continuous variables. What's a circuit? It was enough to simply define a circuit as a collection of electrical components connected by wires, and leave it at that.

\section{Behind the Scenes}

Students in this camp were given a breadboard on which to build digital circuits. Three TTL integrated circuits including eight 2-input NAND gates (two 74LS132s) and six D flip-flops (one 74LS174), and a seven-segment common anode LED display digit with associated currentlimiting resistor were pre-inserted in the breadboard for use in experiments (Figure 1). The

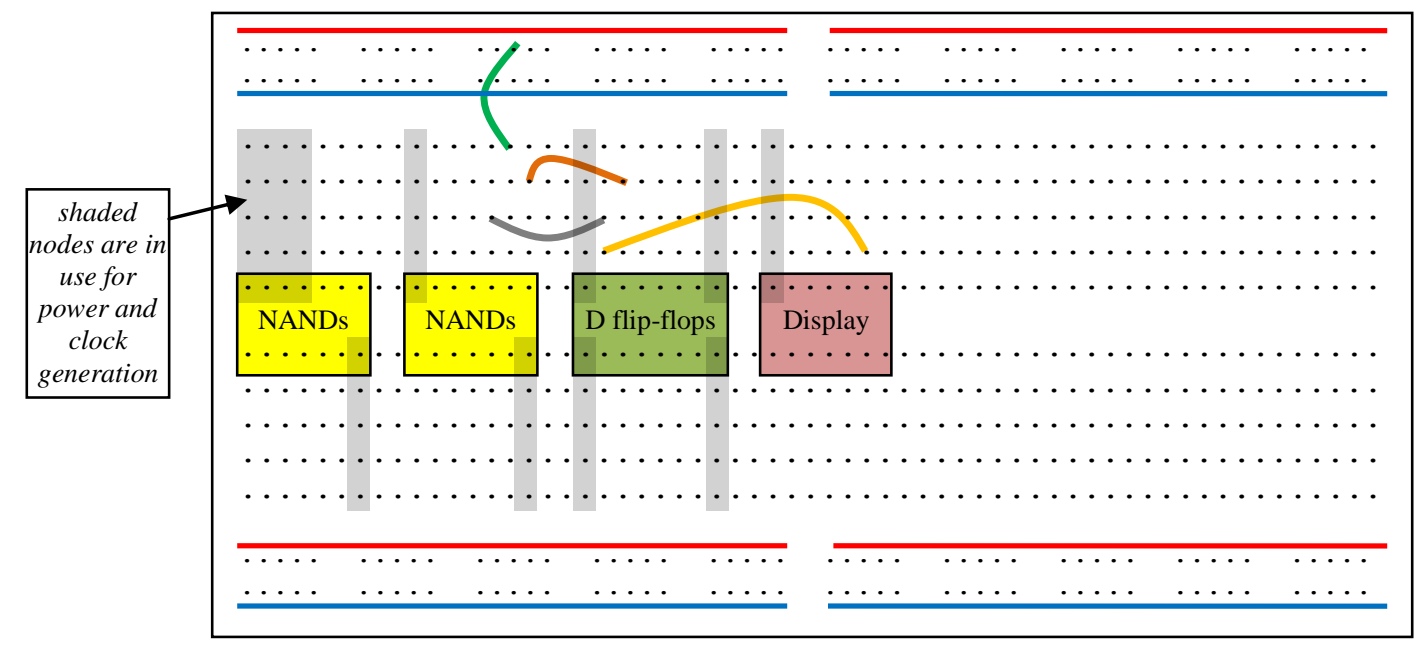

Figure 1: Breadboard with pre-inserted components

NAND gates chosen included hysteresis on the input, i.e. two different switching thresholds based on whether the input voltage was rising or falling. This made possible a very simple clock generation circuit (Figure 2) that was pre-wired on the breadboard for the students. The clock oscillated at about two Hertz and was used as the clock input for the six flip-flops. The clock circuit was not described to the students, as it would have involved some discussion of electrical words such as voltage, current, resistance, capacitance, etc. which we wanted to avoid. Instead, the clock output produced by this circuit was simply another digital signal

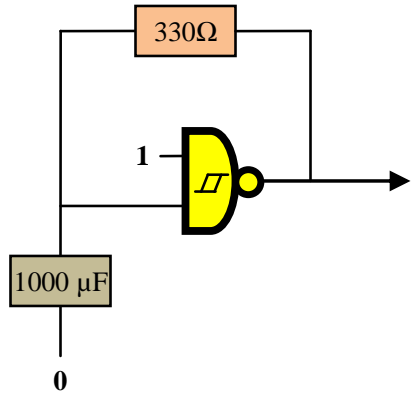

Figure 2: Clock generator to make the flip-flops capture new values and change state every half second. A six-volt lantern battery powered the student circuits. A silicon rectifier diode in series with the battery reduced the voltage to 5.3 volts, safe for TTL.

\section{Combinational Circuits}

The camp described switching algebra (a subset of Boolean algebra using two-valued variables) to students in a simple, intuitive form. AND, OR, and NOT operators were defined, and expressions involving those operators were evaluated using the logic of ordinary English to determine results. No theorems or algebraic manipulations were discussed. The NAND operator was chosen to implement switching functions because it was available with hysteresis inputs (to 
allow the clock generation as described above) and because it is a functionally-complete operator, allowing all switching functions to be implemented with that operator and nothing else, to simplify hardware description.

Functions of only two variables were considered in this camp. Only sixteen two-variable functions exist, and only two of those (exclusive-OR and exclusive-NOR) involve more than one level of gate, ignoring signal inversions. It was easy to explore all sixteen of the possible functions and show how each could be implemented using just NAND gates, sometimes in multiple ways. This encouraged students to realize that digital design, like other types of engineering design, does not have just one answer, often inspiring creativity and ingenuity.

\section{Sequential Circuits}

Sequential circuits, i.e. those including memory so that outputs can depend on the history of circuit operation, require the addition of flip-flops to the digital toolbox. Only D flip-flops were described and installed on the student breadboards, because they are easy to understand and easy to use in designs. Determining the values required on the flip-flop D inputs for given state transitions is intuitive, since the value required on the D input is just whatever the flip-flop is to hold after the next transition on the clock signal. No calculations are needed, just reasoning.

\section{Experiment \#1: Flashing Light}

For the first experiment, a single flip-flop was made to toggle on each clock pulse by simply inverting its output and feeding it back to the D input (Figure 3) so that on each clock the flip-flop captured the complement of the value that it had before the clock. The flip-flop output was displayed on one of the segments of the seven-segment display to produce a flashing light that blinked with a period of about one second. Building this circuit involved adding just

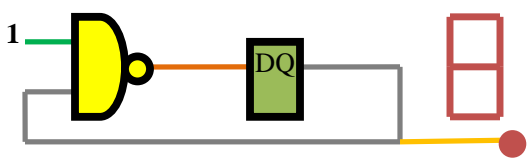

Figure 3: Flashing light four wires to the components that were pre-inserted on the breadboard. The inserted wires are shown in color in Figure 1. This experiment, though simple, gave students a tangible output that brought physical meaning to the topics that were presented.

\section{Experiment \#2: Twisted Ring Counter}

The second experiment expanded the sequential circuit experience by implementing a twelvestate twisted-ring counter (Figure 4). This circuit provides a step-up in complexity of output without requiring a complicated design. In fact, this is really just an extension of the first experiment, replacing the single flip-flop of the first experiment with a chain of six flip-flops. By connecting the six flip-flop outputs to the outer segments of the seven-segment display, and by starting the flip-

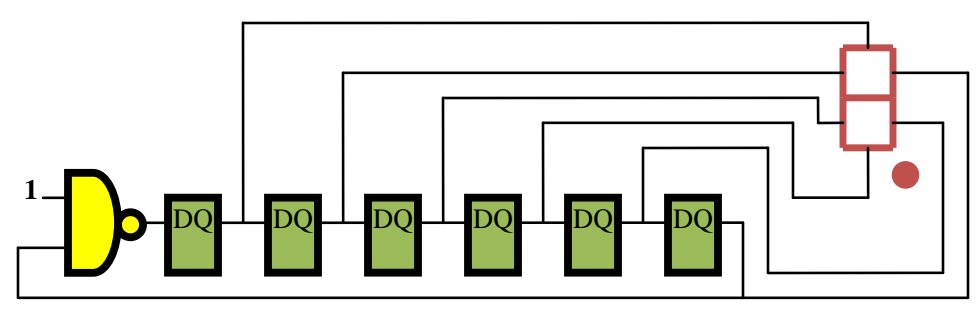

Figure 4: Twelve-state twisted-ring counter 
flops in the all zero's state (by disconnecting the feedback path for a few seconds) the circuit shows a light pattern on the display that circulates counter-clockwise around the outer segments with a period of about six seconds. This was an entertaining result, and demonstrated to the students that seven-segment displays can be used to show things other than just digits zero through nine.

\section{Experiment \#3: Letters on Seven-Segment Display}

Experiment three involved students actually designing their own digital circuits. First, an example was given, using a four-state twisted-ring counter and some NAND logic to display a word, F $\mathbf{u} \mathbf{n}$, on the seven-segment display, one letter per half-second (Figure 5).

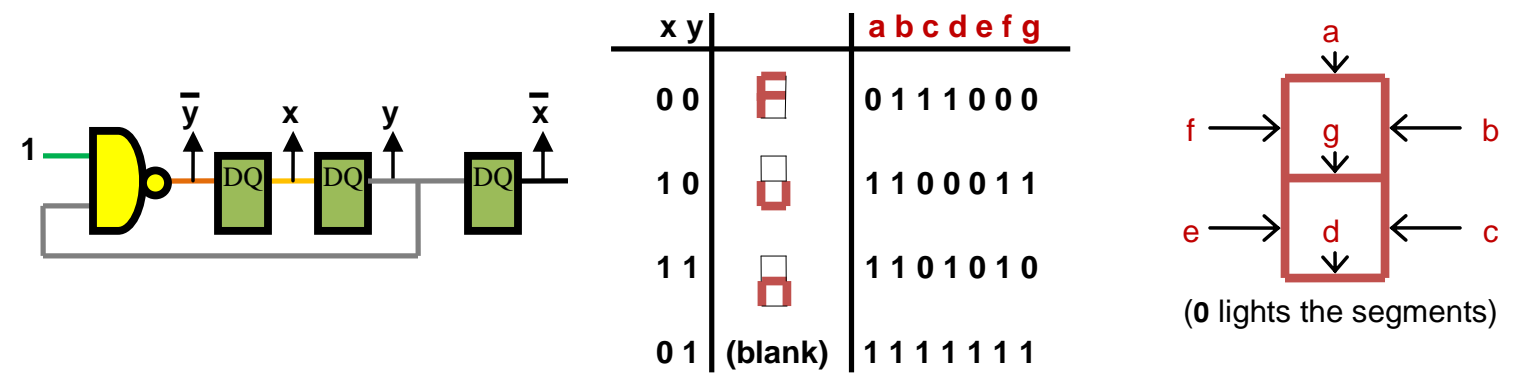

Figure 5: Showing letters on the seven-segment display

The functions for the seven segments of the display to produce this message were analyzed and implemented, in two dramatically different ways (Figure 6).

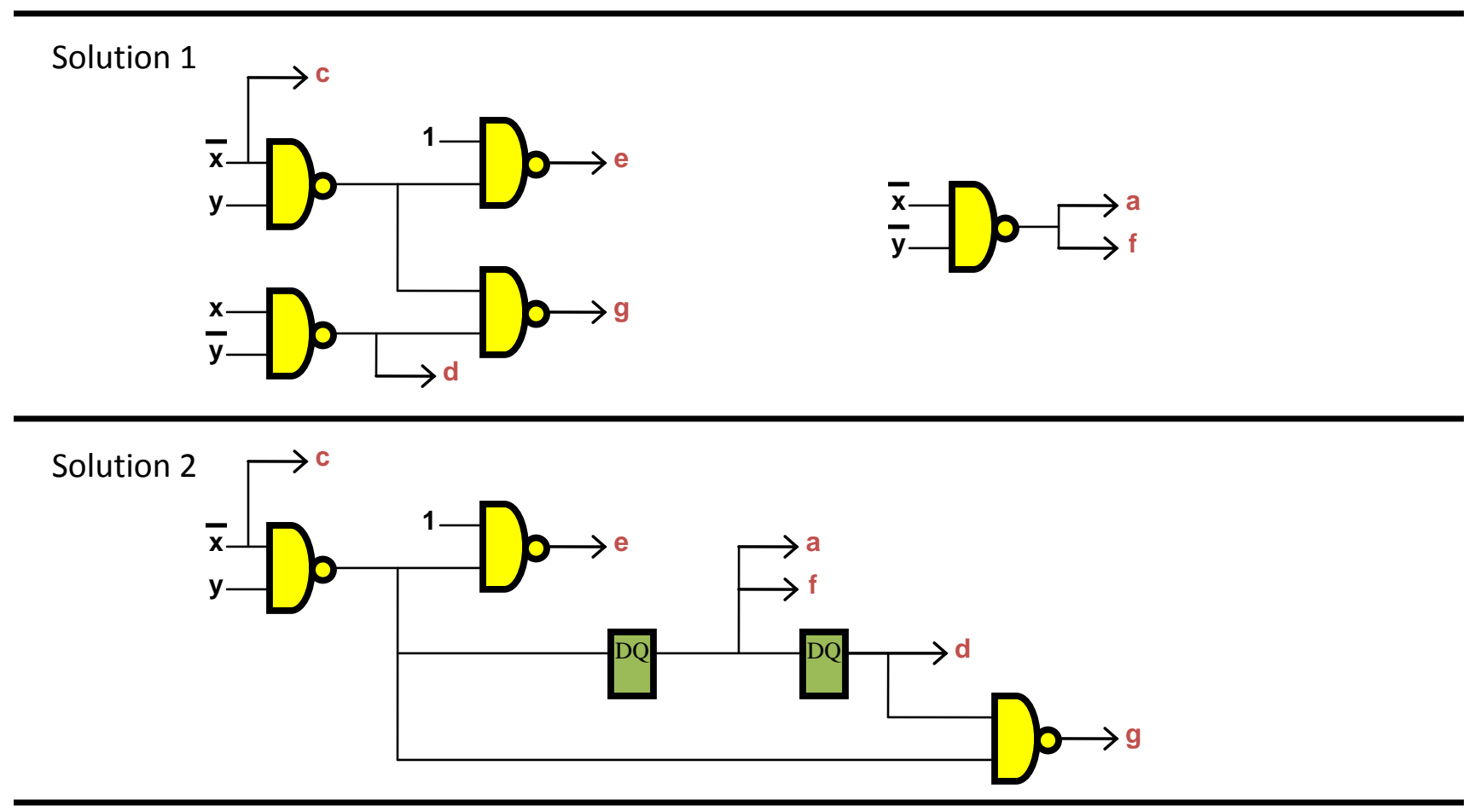

Figure 6: Two dramatically different solutions to "Fun" example 
Then students were asked to pick their own three-letter word and design a circuit to display it in the same way, one letter per half-second, on the seven-segment display, perhaps choosing their initials or any other three-letter word. This was a true design activity, and presented quite a challenge to these middle-school students after only a few hours of experience.

\section{Results}

The one day lesson in digital circuit design during our camp for middle school students seemed to be well-received. Students were surveyed with simple questions to discover their favorite/least favorite activities. Reports from the students' parents indicated that their children enjoyed the experience, and that vocabularies used at home had changed to include some of the terms and expressions that students had heard at camp. The experiments presented to the students challenged them, and pushed them beyond just a cookbook lab experience into the world of true engineering design. They had to consider alternate approaches and select a particular design strategy to develop their own unique solutions. Will these students eventually pursue engineering programs and careers in college and beyond? Only time will tell, but from our perspective the camp succeeded in exposing the students to the excitement of engineering.

\section{Lessons}

The Electrical Engineering faculty presenting this digital circuit design material in camp, who had almost no experience interacting with middle school students, also learned things from the camp. The most important lesson was to avoid long lecture situations. The students were interested and attentive, but fifteen minutes of lecture, no matter how theatrical or entertaining, was the limit. Hands-on activities, such as the lab experiments discussed earlier, were crucial in breaking up the lecture material. These students were active learners, with the emphasis on active! Once we lost their attention, we did not get it back. Of course, engineering topics such as digital circuit design are well-suited to hands-on activities. By starting simply, with the flashing-light experiment, and then building complexity to the point where students were generating independent results, the topic of digital circuit design was effectively presented.

\section{Conclusions}

The goal of this engineering summer camp for middle school students was to interest and inspire participants to consider engineering careers. Digital circuit design, requiring no prerequisites or complicated math, is an excellent vehicle to accomplish that mission. By involving the students in hands-on activities that paralleled topics presented, students started to develop an understanding of the underlying principles behind digital circuits. Students left camp empowered to face today's world of technology with confidence rather than with intimidation.

\section{References}

1. Carroll, C. R., "Summer Camp Exposes Middle-School Girls to Engineering," Proceedings of the 2012 ASEE North Midwest Section Meeting, St. Cloud, MN (2012).

2. Carroll, C. R., "Teaching Ground-Floor Digital Circuits to Pre-Engineering Students," 2011 ASEE Annual Conference, Vancouver, BC (2011). 